\title{
Ultrafast active mixer using polyelectrolytic ion extractor $\dagger$
}

\author{
Honggu Chun, ${ }^{a}$ Hee Chan Kim ${ }^{b}$ and Taek Dong Chung*c
}

\author{
Received 4th October 2007, Accepted 21st February 2008 \\ First published as an Advance Article on the web 11th March 2008 \\ DOI: 10.1039/b715229a
}

We report on a low voltage, straight/smooth surface, and efficient active micromixer. The mixing principle is based on alternative ion depletion-enrichment using a pair of positively charged polyelectrolytic gel electrodes (pPGEs), which face each other joined by a microchannel. This system has an external AC signal source electrically connected to the pPGEs via the respective $1 \mathrm{M} \mathrm{KCl}$ solutions and $\mathrm{Ag} / \mathrm{AgCl}$ electrodes. When an electric bias is applied between the two pPGEs, anions are extracted through one of the pPGEs to create a local ion-deficient region. Simultaneously, an ion-rich area appears near the other pPGE due to an inward anionic flux. As the direction of the charge flow is periodically reversed by the AC signal source, the ion depletion-enrichment regions are alternately swapped with each other on the 'push-pull' basis. The turmoil between the pPGEs quickly mixes the solutions in the microchannel without any mechanical moving part or specially machined structures. In the proposed system, both AC frequency and current density can be easily and finely controlled so that one can quickly find the optimal conditions for a given sample. The micromixer as made showed a mixing efficiency higher than $90 \%$ for sample solutions of $1 \mathrm{mM}$ Rhodamine $6 \mathrm{G}$ and PBS at $\mathrm{pH} 7.4$ when the flow rate was under $6 \mathrm{~mm} \mathrm{~s}^{-1}$. In addition to the solution-solution mixing, the micromixer can effectively mix suspended microparticles with solution. As a representative example, rapid and efficient lysis of human red blood cells was demonstrated allowing minimal damage of the white blood cells.

\section{Introduction}

Microfluidic systems have unique physical features, such as high surface tension and high surface to volume ratio. ${ }^{1}$ Laminar flow is another microfluidic attribute and is due to the low Reynolds number of the fluids in the microchannels (usually $<100$ ). ${ }^{2,3}$ When it comes to quick and efficient mixing, such a laminar flow often gives rise to problems. Since rapid mixing is an essential requirement for many chemical and biological processes, such as studies on the kinetics of enzyme reactions, ${ }^{4}$ RNA folding, ${ }^{5}$ fast liquid-phase reactions, ${ }^{6}$ protein folding, ${ }^{7}$ and cell lysis, ${ }^{8}$ devising a better micromixer has been an issue that is receiving keen attention.

Micromixers can be classified into passive and active types. The former normally involves a complex structure or heterogeneous surface to enhance diffusion or generate chaotic advection. The latter utilizes an external power source to induce turmoil in the fluid. The naive strategy of passive micromixing

\footnotetext{
${ }^{a}$ Department of Chemistry, University of North Carolina, Chapel Hill, NC,USA.E-mail: bioneer@gmail.com; Fax: +1-919-962-4952; Tel: +1-919-962-0272

${ }^{b}$ Department of Biomedical Engineering, College of Medicine and Institute of Medical and Biological Engineering, Medical Research Center, Seoul National University, 28 Yongon-dong, Chongno-gu, Seoul, Korea.E-mail:hckim@snu.ac.kr; Fax: +82-2-2072-7870;

Tel: +82-2-2072-2931

'School of Chemistry, Seoul National University, 56-1 Shilim-dong, Gwanak-gu,Seoul, Korea.E-mail: tdchung@snu.ac.kr; Fax: +82-2-887-4354; Tel: +82-2-880-4354

$\dagger$ Electronic supplementary information (ESI) available: Movie of selective RBC lysis from whole blood sample. See DOI: $10.1039 / \mathrm{b} 715229 \mathrm{a}$
}

is to simply extend the channel length. However, it is a widely held view that long channels are undesirable for miniaturization. In order to increase the contact area between the liquids within a restricted space, various attempts have been made, including multilayer lamination, ${ }^{6,9-18}$ fluid injection, ${ }^{19,20}$ hydrodynamic focusing, ${ }^{7,21}$ nanoporous polymer, ${ }^{22,23}$ and microbead column. ${ }^{24}$ Multilayer lamination and fluid injection reportedly work fairly well, while they require high resolution patterns and complex 2D or 3D structures. The micromixers based on hydrodynamic focusing reduce the width of the sample flow in the middle of sheath flows to minimize the time needed for diffusion. It should be noted that the possible applications of these mixers are substantially limited due to their functions being sensitively affected by the mixing conditions. On the other hand, nanoporous polymers and microbeads for mixing on a microfluidic chip locate themselves in the microfluidic channel to produce random 3D nanofluidic pathways. These micromixers exhibit good mixing performance even within a small dimension. However, they need high pressure pumping sources because nanofluidic pathways along a nanoporous polymer or a microbead column are highly fluid resistive. This may be problematic in sealing and bonding of the microfluidic chip. Moreover, this type of micromixer is inappropriate for mixing large objects such as living cells or microparticles. Another mass transport mode of passive mixing is chaotic advection produced by complex $2 \mathrm{D}$ or $3 \mathrm{D}$ structures, ${ }^{25-31}$ grooves in the channel wall, ${ }^{32-34}$ and heterogeneous surfaces. ${ }^{35-37}$ The $2 \mathrm{D}$ structures used for this purpose include zig-zag-shaped channels ${ }^{27,29}$ and modified Telsa structures. ${ }^{26}$ The zig-zag-shaped channel mixers require a relatively high Reynolds number $(>80)$, whereas the 
mixers based on a modified Telsa structure exhibit good mixing efficiency even at a relatively low Reynolds number ( $>5$ ) owing to the Coanda effect. Reports of $3 \mathrm{D}$ serpentine structures ${ }^{28,31}$ have also been published with various designs, most of which need the Reynolds number to be higher than 10 and the channels longer than $10 \mathrm{~mm}$. Stroock et al. made staggered herringbone patterned grooves on a channel wall to generate chaotic advection and obtained good mixing performance over a wide range of Reynolds numbers $(1<R e<100) .{ }^{34}$ However, this system still requires a long channel $(>10 \mathrm{~mm})$. Johnson et al. proposed a series of ablated wells for another kind of passive mixer. ${ }^{33}$ The surface heterogeneity gave results similar to that of the herringbone grooves. ${ }^{36,37}$ Extending the serpentine microchannel mixer developed by Song et al., ${ }^{38,39}$ Liau et al. reported a droplet-based serpentine microfluidic mixer that induces chaotic mixing of crowded solutions in milliseconds. In this system, the protrusions of the microchannel walls initiate oscillating interfacial shear flow within the droplets. This is useful for kinetics studies but troublesome to perform any subsequent process after mixing, if necessary. ${ }^{40}$ Overall, passive mixers have the essential advantage that no peripheral device, such as an external power source, is required. However, there are a few common drawbacks as well, for example, a high probability of dead volumes trapped at the mixing regions due to their long channels, complicated machining processes required in their production, and difficulty in tuning the mixing condition required for particular samples or flow rates. Futhermore, for cellular applications, their complex structures can impose high sheer stress on cells. Contrary to these passive types, active micromixers can keep their functions under control in most cases. External perturbation sources, such as pressure, ${ }^{41,42}$ thermal, ${ }^{43}$ dielectrophoretic, ${ }^{44,45}$ electrokinetic, ${ }^{46-50}$ magnetic,,${ }^{51,52}$ and acoustic disturbances, ${ }^{53-58}$ induce chaotic advection and instability in the microfluids. Pressure disturbance can produce periodic fluid segmentation or pulsed side flow that leads to instability in the fluid. One of the examples is an integrated thermal bubble pump that constructs wavy interfaces between the fluids. An AC electric field applied through isolated electrodes folds and stretches the interface between the two fluids through dielectrophoretic disturbance. An electrokinetically driven microfluidic mixer is another example using a high AC electrical field $\left(10-100 \mathrm{~V} \mathrm{~mm}^{-1}\right) .{ }^{49}$ It resulted in rapid stirring of two fluid streams under conditions of high voltage $(4-8 \mathrm{kV})$. The switching DC field is grounded in a similar strategy. ${ }^{48}$ Besides the electric field, a rotating magnetic field was applied using an external macro-scale magnetic stirrer ${ }^{51}$ and magnetic beads driven by integrated electromagnets. ${ }^{52}$ Acoustic disturbances generated by integrated piezoelectric transducers are good sources for active mixing as well. ${ }^{54,56,58}$ Consequently, active mixers provide the advantages of rapid mixing ability in short channels and controllable mixing parameters to find the optimal conditions. For all those benefits, they require an external control unit and additional fabrication procedures for electrodes or piezoelectric transducers. In addition, possible cavitation, heat generation and high electric fields may cause problems in biological applications. ${ }^{59}$ In particular, if any mechanical moving part has to be incorporated into the microfluidic system, practical applications that involve cells may be seriously restricted in terms of durability and reliability.
White blood cell (WBC) isolation from a whole blood sample is one of the essential steps in the procedure of single cell analysis including DNA/RNA extraction and protein analysis. A WBC population is separated and thus enriched through selective lysis of red blood cells (RBC). However, WBCs are also hurt under given RBC lysing conditions, such as hypertonic solutions, temperature change, mechanical manipulation, and gradient centrifugation. Long exposure of WBCs to those conditions leads to more serious activation or loss of WBCs. ${ }^{8}$ Therefore, rapid and selective $\mathrm{RBC}$ lysis is crucial to minimize the activation and loss of WBCs. In this respect, any efficient and quick micromixer could result in a successful microfluidic application for RBS lysis.

In this paper, we propose an efficient, active micromixer that is operated with a low voltage, based on the principle of alternating ion depletion-enrichment in the microchannel by employing positively charged polyelectrolytic gel electrodes (pPGEs). The suggested system is sheath-free and has no surface pattern or complicated path involved. We show how it works and the effects of various parameters such as flow rate, switching frequency, and applied potential to the mixing performance. In addition, selective RBC lysis is demonstrated as one biomedical application of the proposed system.

\section{Working principle}

Our previous report showed that the pPGE can play the role of a useful electrode for electrocytometry and velocimetry in a microchannel. ${ }^{60}$ In that study, the pPGEs were fabricated to construct polyelectrolyte plugs that function as good electrical connections between integrated $\mathrm{Ag} / \mathrm{AgCl}$ electrodes, and we emphasized the fact that the chains of the polyelectrolyte bear a stationary charge of high density, like many other ionexchangers, and thus that the charge driven by an external electric field is exclusively carried by counter ions. In a sense, this selective ion transport using pPGE is a similar concept to that of the nanochannel system connected to a microchannel. Wang et al. reported on microfluidic sample preconcentration using an ion depletion phenomenon that can be achieved by an extremely shallow conduit called a nanofluidic channel or nanochannel. ${ }^{61}$ The nanochannel functioned as a one-way pipe for the selective extraction of ions, especially cations in that system. The cation-only flow created an ion depleted region near the entrance of the nanochannel and was applied to realize the novel preconcentration on a microchip. ${ }^{62-64}$ Since the ion transport channels of the pPGEs have the same width and depth as those of the main microchannel, their cross-sectional area should be much larger than that of a nanochannel and thus, the ionic flux becomes far higher. We placed a pair of identical pPGEs facing each other in the direction perpendicular to the main microchannel. As a result, anions come in through one pPGE while escaping from the microchannel through the other at the same rate. That way pPGE can be used to not only extract anions to generate an ion depleted region, but also make anioninjection for local ion enrichment at the same time. In addition, the direction of the ionic flow can be reversed by simply switching the electric bias, resulting in rapid interchange of ion depleted and enriched regions. Wang et al. developed an active mixer in an open chamber using nonlinear electroosmotic microvortices 
caused by a conductive ion-exchange granule. ${ }^{65,66}$ However, the structure limited its application only to stop-flow mixing.

The mechanism of mixing in a microfluidic system using a pPGE pair is illustrated in Fig. 1. Suppose that two solutions flow from left to right in a microchannel in parallel and a pair of pPGEs lie in a channel perpendicular to the main stream, as shown in Fig. 1(a). Fig. 1(b) presents the scheme of what happens
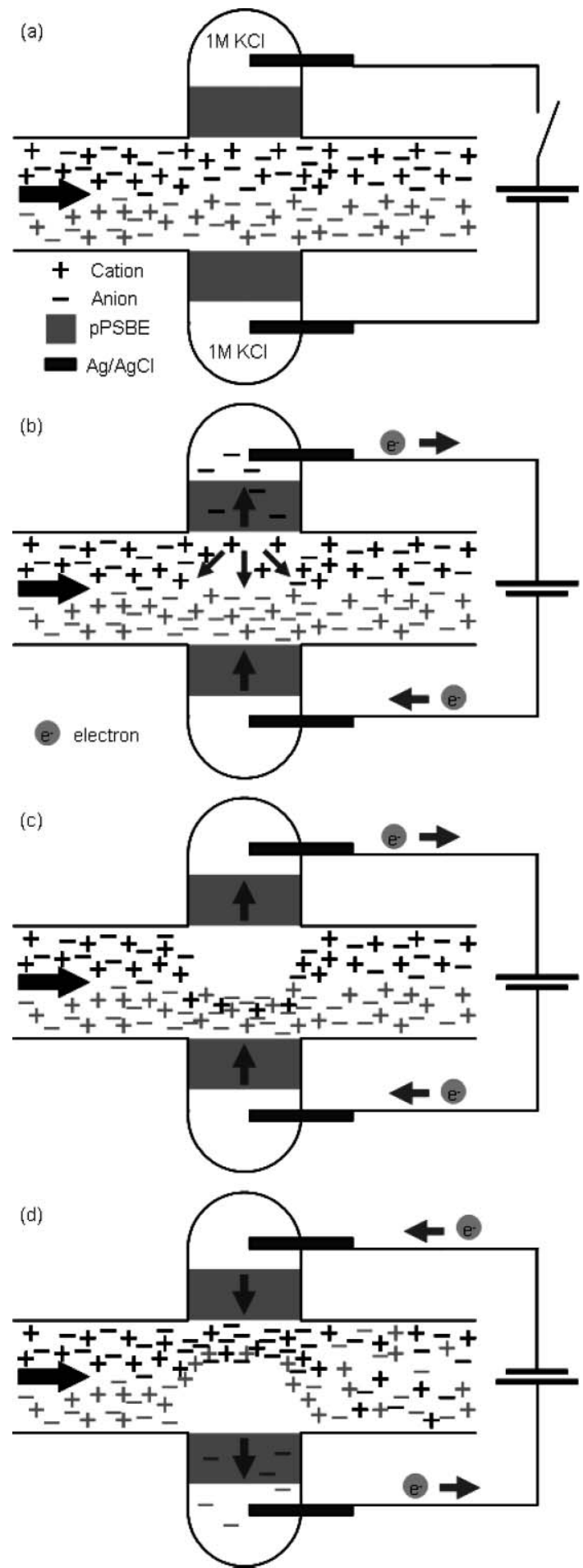

Fig. 1 The mechanism of mixing in a microfluidic system using a pair of pPGEs. when an electric current flows through the pPGE pair. Anions come in through the lower pPGE while going out through the upper one. At the same time, to maintain the electric neutrality, cations are repelled from the region adjacent to the upper $\mathrm{pPGE}$ and simultaneously concentrated near the lower one. As a result, ion depleted and enriched regions appear at the upper and lower parts in the microchannel, respectively (Fig. 1(c)). This heterogeneity in ionic distribution can be actively and quickly driven in microfluids as anionic extraction and injection take place. By reversing the direction of the electric current, the ion depleted and enriched regions are switched, as shown in Fig. 1(d). An AC signal input allows repetitive alternation of ion depletion and enrichment in the local region. For the purpose of mixing, the paired pPGE system is particularly effective because ion depletion as well as ion enrichment simultaneously develops in a narrow mixing spot and the ability to alternate the depletion and enrichment enhances the perturbation effect. Furthermore, an externally applied AC voltage allows fine and handy control of the inward-outward ionic flux rate and the frequency of direction-switching. Therefore, rapid micromixing with excellent efficiency can be realized by incorporating the pPGE system.

\section{Materials and methods}

\section{Chip fabrication}

Corning 2947 precleaned slide glasses $(75 \mathrm{~mm}$ by $25 \mathrm{~mm}, 1 \mathrm{~mm}$ thick) (Corning, NY, USA) were used as substrates. A slide glass was cleaned in a piranha solution $\left(\mathrm{H}_{2} \mathrm{SO}_{4}\right.$ (J.T.Baker, Phillipsburg, NJ, USA) : $\mathrm{H}_{2} \mathrm{O}_{2}(30 \%$, J. T. Baker $\left.)=3: 1\right)$ for $30 \mathrm{~min}$ before washing it with de-ionized (DI) water (NANOpure Diamond, Barnstead, Dubuque, IA, USA) and rinsing with acetone (CMOS grade, J. T. Baker), methanol (CMOS grade, J. T. Baker) and DI water twice, sequentially. The cleaned slide glass was dehydrated on a hot plate (PMC Dataplate 732 , Barnstead) at $120{ }^{\circ} \mathrm{C}$ for $3 \mathrm{~min}$ and cooled to room temperature. Hexamethyl disilazane (HMDS) (Clariant, Switzerland) was spin-coated (Won Corporation, Korea) at $6000 \mathrm{rpm}$ for $30 \mathrm{~s}$ onto the slide glass, which was baked on a hot plate at $120{ }^{\circ} \mathrm{C}$ for $3 \mathrm{~min}$ and then cooled again to room temperature. Then spin-coating of the slide glass with the photo resist (PR) of AZ4620 (Clariant) was subsequently carried out at $6000 \mathrm{rpm}$ for $30 \mathrm{~s}$. After soft baking the PR on a hot plate at $100{ }^{\circ} \mathrm{C}$ for $90 \mathrm{~s}$, the slide glass was cooled to room temperature and then aligned under a pattern mask. Exposing the slide to UV light $(365 \mathrm{~nm})$ with an intensity of $16 \mathrm{~mW} \mathrm{~cm}^{-2}$ for $10.5 \mathrm{~s}$ (MDE-4000, Midas, Korea) was followed by developing the PR with 4 : 1 AZ400K (Clariant) for $5 \mathrm{~min}$. The slide glass was then washed with DI water and the PR was hard-baked on a hot plate at $145^{\circ} \mathrm{C}$ for $15 \mathrm{~min}$. The other side of the slide glass was coated with PR in order to protect it from the etching solution. The slide glass was etched with $6: 1$ buffered oxide etch solution (J. T. Baker) for $40 \mathrm{~min}$ at $25^{\circ} \mathrm{C}$. The washing processes consisted of a few successive steps: rinsing with DI water, sonicating in DI water for $5 \mathrm{~min}$ with an ultrasonic cleaner (3510E-DTH, Bransonic, Danbury, CT, USA), cleaning in piranha solution for $10 \mathrm{~min}$, rinsing with DI water, soaking in acetone, methanol and DI water. Reservoirs with a $1 \mathrm{~mm}$ diameter were drilled with an ultrasonic drill (Gemtech, Korea) on the etched slide glass. 
The etched slide glass and a cover glass $(60 \mathrm{~mm}$ by $24 \mathrm{~mm}$, $100 \mu \mathrm{m}$ thick) (Marienfeld, Germany) were then cleaned in a piranha solution for $1 \mathrm{~h}$ and washed with DI water. The pair, both the etched and cover glasses, were attached by a low temperature bonding technique. A sodium silicate solution (14\% $\mathrm{NaOH}, 27 \% \mathrm{SiO}_{2}$ ) (Sigma-Aldrich, St. Louis, MO, USA) was diluted in DI water (DI water : sodium silicate solution $=5: 1$ ) and then spin-coated onto the cover glass $(1500 \mathrm{rpm}, 7.0 \mathrm{~s})$. Then the pair were immediately brought into contact and annealed on a hot plate at $55^{\circ} \mathrm{C}$ overnight. The microchannel was $70 \mu \mathrm{m}$ wide and $22 \mu \mathrm{m}$ deep.

\section{Safety precautions}

Piranha solution is a powerful oxidizer and reacts violently with organic materials or solvents and should be handled with extreme care.

\section{pPGE fabrication}

The pPGE fabrication techniques are based on our previous work. ${ }^{60}$ Briefly, diallyldimethylammonium chloride (DADMAC) was chosen as the monomer for the polyelectrolytic polymer plugs. A $65 \%$ DADMAC aqueous solution (Sigma-Aldrich) was polymerized to yield polyDADMAC (PDADMAC) by exposing UV light in the presence of $2 \%$ photo-initiator (2-hydroxy-4'-(2-hydroxyethoxy)-2methylpropiophenone) (Sigma-Aldrich) and 2\% cross linker ( $N, N$ '-methylene-bisacrylamide) (Sigma-Aldrich). In this study, a coating procedure prior to photopolymerization was added to the fabrication process in order to covalently link PDADMAC to the channel. TMSMA (3-(Trimethoxysilyl)propyl methacrylate) (Sigma-Aldrich) was diluted in methanol at a concentration of $0.1 \mathrm{M}$. The fabricated glass chip was filled with the diluted TMSMA solution and sealed with parafilm for $1 \mathrm{~h}$. After that, the chip was washed with methanol. Then, the pPGE was fabricated as follows. The microchannel network of a microfluidic glass chip was filled with DADMAC solution of the composition described above. The chip was aligned under the mask and subsequently exposed to UV light $(365 \mathrm{~nm})$ at $16 \mathrm{~mW} \mathrm{~cm} \mathrm{~cm}^{-2}$ for $4.0 \mathrm{~s}$. Then, the DADMAC monomers were polymerized to form PDADMAC immobilized on the lateral channel walls. After photopolymerization, the microchannel was cleaned with a $1 \mathrm{M} \mathrm{KCl}$ solution to remove the remaining DADMAC monomers.

\section{Experimental setup}

Fig. 2 shows the configuration of the microfluidic glass chip on which the pPGE-active mixer works. A pair of pPGEs were formed at the two lateral branches perpendicular to the main microchannel and were connected to an external AC signal source (AFG310, Tektronix, Richardson, TX, USA) via their respective $1 \mathrm{M} \mathrm{KCl}$ solutions and $\mathrm{Ag} / \mathrm{AgCl}$ electrodes. A rectangular wave AC signal was applied between the two $\mathrm{Ag} / \mathrm{AgCl}$ electrodes. Sample solutions were injected into the microchannel using a syringe pump (KDS120, KD Scientific, Holliston, MA, USA). The experimental tests were performed on an inverted fluorescence microscope (TE1200-U, Nikon, Japan). Images were acquired with a CCD module with the

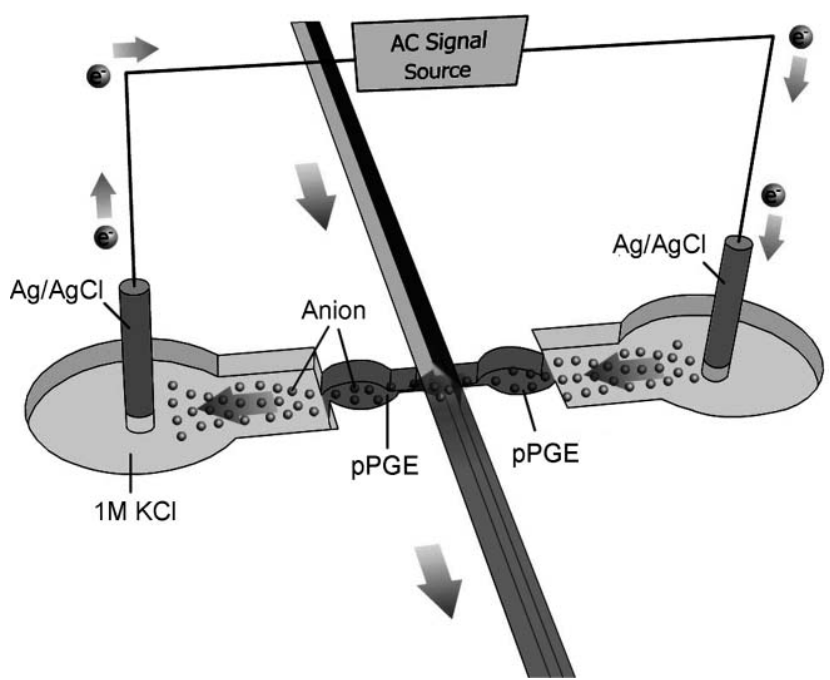

Fig. 2 Active mixing in a microchannel with pPGEs. A rectangular wave $\mathrm{AC}$ signal was applied between two $\mathrm{Ag} / \mathrm{AgCl}$ electrodes, which were electrochemically connected to two pPGEs through $1 \mathrm{M} \mathrm{KCl}$ solution.

resolution of 720 by $480,125^{-1}$ s shutter speed, at 30 frames per second.

\section{Experimental procedure}

The performance of the active mixing microchip with pPGEs was evaluated by measuring the fluorescent intensity. The effect of AC amplitude on the mixing efficiency was investigated by varying the potential from $0.5 \mathrm{~V}$ to $10 \mathrm{~V}$. The frequency effect was examined by setting the frequency from $1 \mathrm{~Hz}$ to $100 \mathrm{~Hz}$. The flow rate effect was probed by checking the mixing efficiency for the flow rate from 0 to $33 \mathrm{~mm} \mathrm{~s}^{-1}$. A mixing efficiency parameter, $\varepsilon(x)$, can be defined as ${ }^{36}$

$$
\varepsilon(x)=\left(1-\frac{\int\left|C(x, y)-C_{\infty}\right| \mathrm{d} y}{\int\left|C_{0}-C_{\infty}\right| \mathrm{d} y}\right) \times 100 \%
$$

where $C(x, y)$ is the cross sectional concentration profile at a distance $x$ downstream of a mixer. $C_{\infty}$ and $C_{0}$ are the concentration profiles associated with completely mixed and completely unmixed states, respectively. The concentration distribution data were acquired $100 \mu \mathrm{m}$ downstream $(x=100 \mu \mathrm{m})$ from the end of pPGE. The regions directly adjacent to the channel walls were not included in the analysis ${ }^{22,67}$ because the microchannel was wet etched, and therefore did not have a sharp edge profile. This procedure was performed for $80 \%$ of the full width of the cross section.

The performance was also evaluated with fluorescent polystyrene latex microbeads (Fluoresbrite ${ }^{\mathrm{TM}}$ plain YG $6.0 \mathrm{mi}-$ cron microspheres, Polysciences, Warrington, PA, USA). The microbeads were $5.70 \mu \mathrm{m}$ in diameter on average (standard deviation $=0.219 \mu \mathrm{m}$ ), which is similar to that of a human red blood cell. The fluorescent microbeads were diluted in $1 \mathrm{mM}$ PBS at pH 7.4 to $2.1 \times 10^{7}$ particles per $\mathrm{mL}$ just before use.

Finally, the mixer was applied to selective RBC lysis. The human blood was sampled from a healthy subject in an EDTA treated tube (BD Vacutainer, K2 EDTA 7.2mg, BD, Franklin 
Lakes, NJ, USA), and was then diluted in isotonic PBS at $\mathrm{pH} 7.4$ to $1: 9$. WBCs were selectively stained with calcein. Then, $2 \mu \mathrm{L}$ of $1 \mathrm{mg} \mathrm{mL}^{-1}$ calcein-AM solution in anhydrous DMSO (Invitrogen, Carlsbad, CA, USA) was added to $20 \mu \mathrm{L}$ of diluted whole blood. The mixed solution was incubated for $15 \mathrm{~min}$ at $37^{\circ} \mathrm{C}$ for staining. RBC lysing solution was prepared by diluting Zap-oglobin $^{\mathrm{TM}}$ II Lytic Reagent (Beckman Coulter, Fullerton, CA, USA) in isotonic PBS at $\mathrm{pH} 7.4$ to $1: 40$.

\section{Results and discussion}

\section{Mixing of fluorescent solutions}

Fig. 3 shows ion depletion and enrichment phenomena caused by selective ion transfer through the pPGE. $1 \mathrm{mM}$ PBS (pH 7.4) and $100 \mu \mathrm{M}$ Rhodamine $6 \mathrm{G}$ (positively charged at this $\mathrm{pH}$ ) solution were allowed to flow in parallel along the microchannel inlets from left to right. Initially, the rectangular wave AC source was turned off. Rhodamine $6 \mathrm{G}$ and PBS solutions form a laminar flow throughout the whole channel, as indicated in Fig. 3(a) When the AC source was turned on, the upper pPGE and lower pPGE became the anion injector and extractor, respectively. Rhodamine $6 \mathrm{G}$, a positively charged dye, started to be depleted near the lower pPGE and the transparent region expanded from the pPGE interface. This indicates that an ion depletion region had been created, as shown in Fig. 3(b)
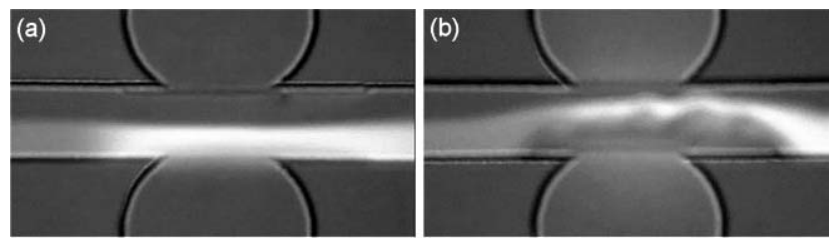

Fig. 3 The mixing procedure using pPGEs. A $100 \mu \mathrm{M}$ Rhodamine $6 \mathrm{G}$ solution and $1 \mathrm{mM}$ PBS at $\mathrm{pH} 7.4$ formed a laminar flow when the mixer was turned off (a). By applying $5 \mathrm{~V}$ between the pPGEs as the lower pPGE positive, anions were injected and extracted through the upper and lower pPGE, respectively. Then, ion depletion and enrichment regions were created (b).

By alternating the polarity, the location of ion depletionenrichment regions were correspondingly switched, resulting in turmoil between the pPGEs and a mixing of the two solutions. When an alternating frequency of $10 \mathrm{~Hz}$ was applied, the mixing process was completed at $100 \mu \mathrm{m}$ downstream from the pPGEs, as displayed in Fig. 4(a) and Fig. 4(b).

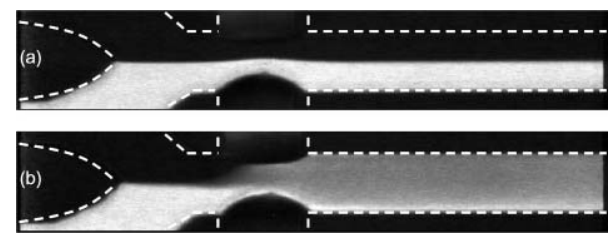

Fig. 4 A $100 \mu \mathrm{M}$ Rhodamine $6 \mathrm{G}$ solution and $1 \mathrm{mM}$ PBS at $\mathrm{pH} 7.4$ were injected to the lower and upper Y channel inlet, respectively. The two solutions flowed through the main microchannel from left to right. (a) Mixer off. The two solutions formed a stable laminar flow. (b) Mixer on with $10 \mathrm{~Hz}$ of alternating frequency. Upon $5 \mathrm{~V}$ of applied potential, the two solutions were mixed very rapidly.
The frequency of the applied AC potential was predicted to affect the mixing efficiency. If the frequency was too high, the polarity of each pPGE would inverted without enough duration for ion extraction and injection. On the other hand, when the frequency was too low, the solutions would pass through the mixing region before sufficient mixing had taken place. Therefore both extremes would result in low mixing efficiency. Fig. 5(a) shows the variation of mixing efficiency for the applied
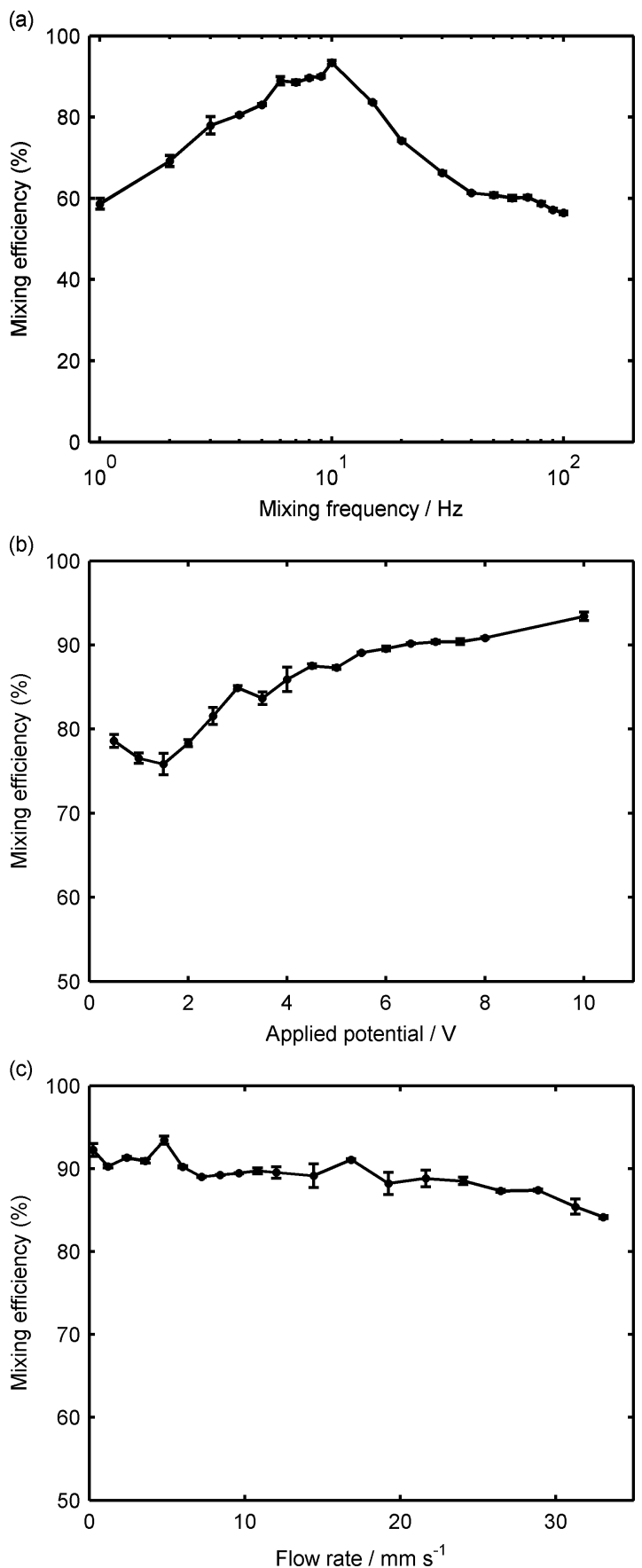

Fig.5 Dependence of mixing efficiency on frequency, applied potential, and flow rate. (a) The frequency effect. The mixing efficiency reached its maximum at $10 \mathrm{~Hz}$. (b) The effect of an applied potential between two $\mathrm{Ag} / \mathrm{AgCl}$ electrodes. (c) The flow rate effect. The mixing efficiency gradually decreased as the flow rate was increased. 
frequency from 1 to $100 \mathrm{~Hz}$ with the flow rate, the applied potential, and the concentration of $5 \mathrm{~mm} \mathrm{~s}^{-1}, 10 \mathrm{~V}$, and $1 \mathrm{mM}$, respectively. The mixing efficiency reached its maximum when the applied frequency was around $10 \mathrm{~Hz}$. When the applied frequency was lower or higher than $10 \mathrm{~Hz}$, the mixing efficiency notably dropped. Mixing efficiency is also a function of the applied potential between the two $\mathrm{Ag} / \mathrm{AgCl}$ electrodes. It was expected that higher potential would drive faster ion extraction and injection, leading to more efficient mixing. The mixing efficiencies were recorded as the applied potential varied from 0.5 to $10 \mathrm{~V}$ with the flow rate, sample concentration and the applied frequency of $5 \mathrm{~mm} \mathrm{~s}^{-1}, 1 \mathrm{mM}$, and $10 \mathrm{~Hz}$, respectively. The results in Fig. 5(b) show that the mixing efficiency is a function of the applied voltage and the mixer operates efficiently with low voltage under $10 \mathrm{~V}$. The flow rate may affect the mixing efficiency. If the flow rate is high, the system will not allow enough time for the solutions to be mixed. The flow rate was varied from 0.01 to $33 \mathrm{~mm} \mathrm{~s}^{-1}$ with the applied potential, the applied frequency, and the sample concentration at $10 \mathrm{~V}, 10 \mathrm{~Hz}$, and $1 \mathrm{mM}$, respectively. Fig. 5(c) shows that the mixing efficiency decreases slightly as the flow rate increases.

\section{Mixing of microparticles}

The proposed pPGE ion extractor system can work to mix nano- or microparticles with reagents in the microfluidic system efficiently and quickly. In order to verify this, fluorescent polystyrene latex microbeads of $5.70 \mu \mathrm{m}$ in diameter were dispersed in $1 \mathrm{mM}$ PBS at pH 7.4 and were mixed with microbeadfree PBS. Fig. 6 shows streaks of fluorescent microbeads indicating their trajectories. A sample solution containing fluorescent microbeads was injected into the lower arm of the Y-shaped horizontal microchannel. The flow rate in the main microchannel was $6 \mathrm{~mm} \mathrm{~s}^{-1}$. The trajectories of the microbeads showed a laminar flow until the mixer was turned on, as shown in Fig. 6(a). When the mixer was turned on by applying an $20 \mathrm{~Hz}$ AC source of $10 \mathrm{~V}$, anions started to be depleted and be injected through the pPGEs, and alternating ion depletion-enrichment regions were made between the pPGEs with corresponding AC frequency. This ion movement generated sufficient turmoil to mix the microbeads with the solution within a short distance, as shown in Fig. 6(b) The fluorescent microbeads in this study were of a similar size to that of human blood cells.
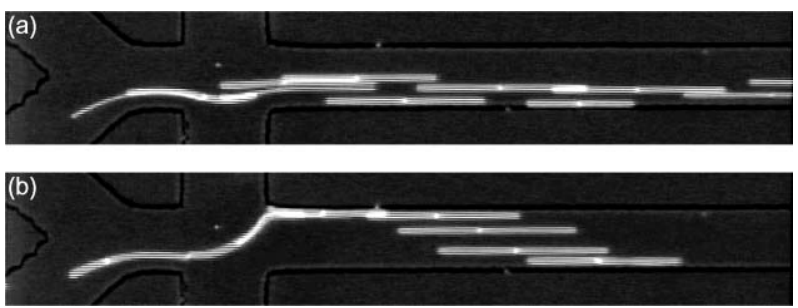

Fig. 6 Mixing fluorescent microbeads with an aqueous solution. Fluorescent polystyrene latex microbeads of comparable size with cells were used. The trajectories showed a laminar flow when the mixer was turned off (a). By applying $20 \mathrm{~Hz} \mathrm{AC}$ source of $10 \mathrm{~V}$, microbeads were successfully mixed (b).

\section{RBC lysis}

Finally, the mixer was applied to selective RBC lysis. A diluted whole blood sample containing fluorescent labeled WBCs was quickly mixed with lysing solution. In Fig. 7(a), a blood sample was injected into the lower arm of the Y-shaped horizontal microchannel at a flow rate of $300 \mu \mathrm{m} \mathrm{s}^{-1}$. When a $20 \mathrm{~Hz}$ AC source of $6 \mathrm{~V}$ was applied, RBCs in the sample passing through the mixing region were completely lysed, and therefore, there were no RBC in the downstream channel and only WBC travelled downstream, as shown in Fig. 7(b), Fig. 7(c), and Fig. 7(d) (see ESI $\dagger$ ). The RBCs were lysed in less than $1 \mathrm{~s}$. We repeated the same experiment after replacing the lysing solution with isotonic PBS at $\mathrm{pH}$ 7.4. While the blood sample was mixed with the PBS, no lysing event was observed. The pPGE-based micromixer proposed in this study showed quick RBC-selective lysis, which strongly suggests that WBC damage can be minimized by combining with a WBC collector.
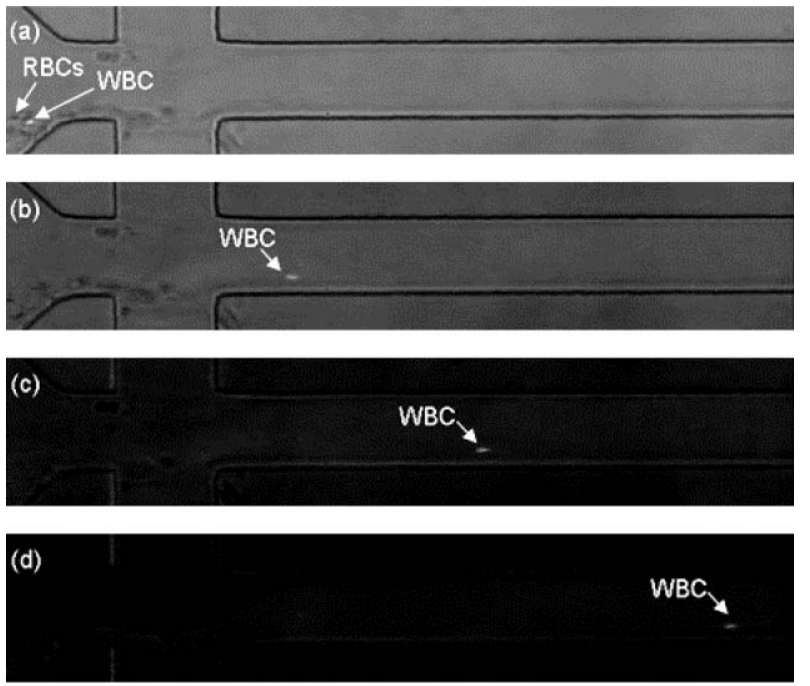

Fig. 7 Selective RBC lysis from whole blood sample. (a) RBCs and fluorescent labeled WBCs were flowing through the channel. (b) When they were passing the mixing region, RBCs started to lyse, but (c) WBCs passed through the region. (d) Only WBCs flowed downstream.

\section{Conclusions}

An active mixing method based on alternating ion depletionenrichment using pPGEs was developed and its performance was evaluated. Owing to the large cross sectional area of the pPGE, sufficient mixing was achieved within about $100 \mu \mathrm{m}$ from the pPGE, which is suited to microchip applications. The results confirmed that this method satisfactorily provides mixing efficiency under reasonable conditions. The applicability of the proposed mixer to mixing microparticles with aqueous reagents was successfully verified. Moreover, it was successfully applied to rapid and selective RBC lysis; therefore, it is expected that this type of micromixer may expand its range to biological applications requiring quick mixing of cell and reagents in microfluidic systems.

The pPGEs are chemically stable. If the microchip with pPGEs is stored in a $1 \mathrm{M} \mathrm{KCl}$ solution, the pPGEs keep their mechanical and electrical properties intact over one year. Furthermore, 
the polyelectrolytic plugs maintain their initial shape and no swelling is observed. There is no leakage through the pPGE because the polymer plugs are tightly bound to the microchannel by covalent bonds. Therefore, stopped flow mixing is possible, which is unattainable by passive mixers.

Ion transport across the pPGEs is so facile that the electrical conductance is sufficiently high that a low voltage power supply is enough to operate the proposed micromixer, a circumstance almost unrealisable for previously reported electrokinetic mixers. Besides, the structure of the proposed mixer using pPGEs is simple, and easy to fabricate and handle because this device involves no active mechanical parts, and thus the system is mechanically robust. All these features allow us to expect that the proposed system will be a competitive alternative to conventional mixing methods.

\section{Acknowledgements}

This work was supported by the Korea Science and Engineering Foundation (KOSEF) grant funded by the Korea government (MOST) (No. R11-2007-012-02002-0) and by the MKE (Ministry of Knowledge Economy), Korea, under the ITRC (Information Technology Research Center) support program supervised by the IITA (Institute of Information Technology Advancement) (IITA-2008-(C1090-0801-0002)).

\section{Notes and references}

1 D. J. Beebe, G. A. Mensing and G. M. Walker, Annu. Rev. Biomed. Eng., 2002, 4, 261-286.

2 P. Gravesen, J. Branebjerg and O. S. Jensen, J. Micromech. Microeng., 1993, 3, 168-182.

3 P. J. A. Kenis, R. F. Ismagilov and G. M. Whitesides, Science, 1999, 285, 83-85.

4 L. Luo, M. D. Burkart, T. Stachelhaus and C. T. Walsh, J. Am. Chem. Soc., 2001, 123, 11208-11218.

5 X. W. Fang, P. Thiyagarajan, T. R. Sosnick and T. Pan, Proc. Natl. Acad. Sci. U. S. A., 2002, 99, 8518-8523.

6 T. M. Floyd, M. A. Schmidt and K. F. Jensen, Ind. Eng. Chem. Res., 2005, 44, 2351-2358.

7 D. E. Hertzog, X. Michalet, M. Jager, X. Kong, J. G. Santiago, S. Weiss and O. Bakajin, Anal. Chem., 2004, 76, 7169-7178.

8 P. Sethu, M. Anahtar, L. L. Moldawer, R. G. Tompkins and M. Toner, Anal. Chem., 2004, 76, 6247-6253.

9 J. Branebjerg, P. Gravesen, J. P. Krog and C. R. Nielsen, in Micro Electro Mechanical Systems, MEMS '96, Proceedings. 'An Investigation of Micro Structures, Sensors, Actuators, Machines and Systems'. IEEE, The Ninth Annual International Workshop, San Diego, CA, USA, 1996, pp. 441-446.

10 B. J. Burke and F. E. Regnier, Anal. Chem., 2003, 75, 1786-1791.

11 W. Ehrfeld, K. Golbig, V. Hessel, H. Lowe and T. Richter, Ind. Eng. Chem. Res., 1999, 38, 1075-1082.

12 C. Erbacher, F. G. Bessoth, M. Busch, E. Verpoorte and A. Manz, Microchim. Acta, 1999, 131, 19-24.

13 B. He, B. J. Burke, X. Zhang, R. Zhang and F. E. Regnier, Anal. Chem., 2001, 73, 1942-1947.

14 M. Kakuta, F. G. Bessoth and A. Manz, Chem. Rec., 2001, 1, 395 405.

15 M. Koch, D. Chatelain, A. G. R. Evans and A. Brunnschweiler, J. Micromech. Microeng., 1998, 8, 123-126.

16 H. Mensinger, T. Richer, V. Hessel, J. Dopper and W. Ehrfeld, in Proc. Micro Total Anal. Syst. Workshop, Enschede, The Netherlands, 1994, pp. 237-243.

17 M. S. Munson and P. Yager, in Proc. 7th Int. Conf. Miniaturized Chem. Biochem. Anal. Syst., 2003, pp. 495-498.

18 N. Schwesinger, T. Frank and H. Wurmus, J. Micromech. Microeng., 1996, 6, 99-102.
19 K. Ke, J. Ernest, F. Hasselbrink and A. J. Hunt, Anal. Chem., 2005, 77, 5083-5088.

20 R. Miyake, T. S. J. Lammerink, M. Elwenspoek and J. H. J. Fluitman, in Micro Electro Mechanical Systems, MEMS '93, Proceedings 'An Investigation of Micro Structures, Sensors, Actuators, Machines and Systems', IEEE., 1993, pp. 248-253.

21 J. B. Knight, A. Vishwanath, J. P. Brody and R. H. Austin, Phys. Rev. Lett., 1998, 80, 3863-3866.

22 S. Jeon, V. Malyarchuk, J. O. White and J. A. Rogers, Nano Lett., 2005, 5, 1351-1356.

23 T. Rohr, C. Yu, M. H. Davey, F. Svec and J. M. J. Frechet, Electrophoresis, 2001, 22, 3959-3967.

24 G. H. Seong, W. Zhan and R. M. Crooks, Anal. Chem., 2002, 74, 3372-3377.

25 H. Aref, Phys. Fluids, 2002, 14, 1315-1325.

26 C.-C. Hong, J.-W. Choi and C. H. Ahn, Lab Chip, 2004, 4, 109-113.

27 S. W. Jones, O. M. Thomas and H. Aref, J. Fluid Mech., 1989, 209, 335-357.

28 R. H. Liu, M. A. Stremler, K. V. Sharp, M. G. Olsen, J. G. Santiago, R. J. Adrian, H. Aref and D. J. Beebe, J. Microelectromech. Syst., 2000, 9, 190-197.

29 V. Mengeaud, J. Josserand and H. H. Girault, Anal. Chem., 2002, 74, 4279-4286.

30 F. Schonfeld, V. Hessel and C. Hofmann, Lab Chip, 2004, 4, 65-69.

31 D. Therriault, S. R. White and J. A. Lewis, Nat. Mater., 2003, 2, 265-271.

32 J. T. Cabral, S. D. Hudson, C. Harrison and J. F. Douglas, Langmuir, 2004, 20, 10020-10029.

33 T. J. Johnson, D. Ross and L. E. Locascio, Anal. Chem., 2002, 74, 45-51.

34 A. D. Stroock, S. K. W. Dertinger, A. Ajdari, I. Mezic, H. A. Stone and G. M. Whitesides, Science, 2002, 295, 647-651.

35 E. Biddiss, D. Erickson and D. Li, Anal. Chem., 2004, 76, 3208-3213.

36 D. Erickson and D. Li, Langmuir, 2002, 18, 1883-1892.

37 A. D. Stroock, M. Weck, D. T. Chiu, W. T. S. Huck, P. J. A. Kenis, R. F. Ismagilov and G. M. Whitesides, Phys. Rev. Lett., 2000, 84, 3314-3317.

38 H. Song, J. D. Tice and R. F. Ismagilov, Angew. Chem., Int. Ed., 2003, 42, 768-772.

39 H. Song and R. F. Ismagilov, J. Am. Chem. Soc., 2003, 125, 1461314619.

40 A. Liau, R. Karnik, A. Majumdar and J. H. D. Cate, Anal. Chem., 2005, 77, 7618-7625.

41 A. A. Deshmukh, D. Liepmann and A. P. Pisano, in IEEE Proc. Solid State Sens. Actuator Workshop, 2000, pp. 73-76.

42 T. Thorsen, S. J. Maerkl and S. R. Quake, Science, 2002, 298, 580584.

43 J.-H. Tsai and L. Lin, Sens. Actuators, A, 2002, 97-98, 665-671.

44 J. Deval, P. Tabeling and C.-M. Ho, in Micro Electro Mechanical Systems, The Fifteenth IEEE International Conference, 2002, pp. 36-39.

45 Y.-K. Lee, J. Deval, P. Tabeling and C.-M. Ho, in Micro Electro Mechanical Systems, MEMS 2001, The 14th IEEE International Conference, Interlaken, Switzerland, 2001, pp. 483-486.

46 P. Huang and K. S. Breuer, in TRANSDUCERS, Solid-State Sensors, Actuators and Microsystems, 12th IEEE International Conference, 2003, pp. 663-666.

47 S. C. Jacobson, T. E. McKnight and J. M. Ramsey, Anal. Chem., 1999, 71, 4455-4459.

48 C.-H. Lin, L.-M. Fu and Y.-S. Chien, Anal. Chem., 2004, 76, 52655272.

49 M. H. Oddy, J. G. Santiago and J. C. Mikkelsen, Anal. Chem., 2001, 73, 5822-5832

50 H. Y. Wu and C. H. Liu, in IEEE Proc. Solid State Sens. Actuator Workshop, 2003, pp. 631-634.

51 L.-H. Lu, K. S. Ryu and C. Liu, J. Microelectromech. Syst., 2002, 11, 462-469.

52 R. Rong, J. W. Choi and C. H. Ahn, in Proc. 7th Int. Conf. Miniaturized Chem. Biochem. Anal. Syst., 2003, pp. 335-338.

53 R. H. Liu, R. Lenigk, R. L. Druyor-Sanchez, J. Yang and P. Grodzinski, Anal. Chem., 2003, 75, 1911-1917.

54 J. C. Rife, M. I. Bell, J. S. Horwitz, M. N. Kabler, R. C. Y. Auyeung and W. J. Kim, Sens. Actuators, A, 2000, 86, 135-140.

55 T. R. Tsao, R. M. Moroney, B. A. Martin and R. M. White, in Ultrasonics Symposium, Proceedings., IEEE, 1991, pp. 937-940. 
56 V. Vivek and E. S. Kim, in Micro Electro Mechanical Systems, MEMS 2000, The Thirteenth IEEE Annual International Conference on, 2000, pp. 668-673.

57 Z. Yang, S. Matsumoto, H. Goto, M. Matsumoto and R. Maeda, Sens. Actuators, A, 2001, 93, 266-272.

58 G. G. Yaralioglu, I. O. Wygant, T. C. Marentis and B. T. KhuriYakub, Anal. Chem., 2004, 76, 3694-3698.

59 N.-T. Nguyen and Z. Wu, J. Micromech. Microeng., 2005, 15, 1-16.

60 H. Chun, T. D. Chung and H. C. Kim, Anal. Chem., 2005, 77, 2490 2495.

61 Y.-C. Wang, A. L. Stevens and J. Han, Anal. Chem., 2005, 77, $4293-$ 4299.
62 A. Plecis, R. B. Schoch and P. Renaud, in MicroTAS, Boston, Massachusetts, USA, 2005, pp. 1038-1041.

63 Q. S. Pu, J. S. Yun, H. Temkin and S. R. Liu, Nano Lett., 2004, 4, 1099-1103.

64 S. J. Kim, Y.-C. Wang, H. Jang, J. H. Lee and J. Han, in MicroTAS, Tokyo, Japan, 2006, pp. 522-524.

65 S.-C. Wang, Y.-W. Lai, Y. Ben and H.-C. Chang, Ind. Eng. Chem. Res., 2004, 43, 2902-2911.

66 S.-C. Wang, H.-P. Chen, Y.-W. Lai, L.-K. Chau, Y.-C. Chuang and Y.-J. Chen, Biomicrofluidics, 2007, 1, 034104.

67 J. Voldman, M. L. Gray and M. A. Schmidt, J. Microelectromech. Syst., 2000, 9, 295-302. 